\title{
Valérian GEFFROY, Des spatialités qui engagent et qui lient : le tourisme sportif de nature et ses communautés de pratique
}

Thèse de doctorat en géographie, Université de Lausanne, sous la direction de Mathis Stock, soutenue le 6 mars 2020

Spatialities that engage and bond: outdoor sports tourism and its practice communities

\section{Valérian Geffroy}

\section{OpenEdition Journals}

Édition électronique

URL : http://journals.openedition.org/tourisme/2917

DOI : $10.4000 /$ tourisme.2917

ISSN : 2492-7503

Éditeur

Éditions touristiques européennes

\section{Référence électronique}

Valérian Geffroy, «Valérian GeFFroy, Des spatialités qui engagent et qui lient : le tourisme sportif de nature et ses communautés de pratique », Mondes du Tourisme [En ligne], 17 | 2020, mis en ligne le 01 juin 2020, consulté le 20 décembre 2020. URL : http://journals.openedition.org/tourisme/2917; DOI : https://doi.org/10.4000/tourisme.2917

Ce document a été généré automatiquement le 20 décembre 2020.

\section{(c)}

Mondes du tourisme est mis à disposition selon les termes de la licence Creative Commons Attribution - Pas d'Utilisation Commerciale - Pas de Modification 4.0 International. 


\section{Valérian GEFFROY, Des spatialités qui engagent et qui lient : le tourisme sportif de nature et ses communautés de pratique}

Thèse de doctorat en géographie, Université de Lausanne, sous la direction de Mathis Stock, soutenue le 6 mars 2020

Spatialities that engage and bond: outdoor sports tourism and its practice communities

Valérian Geffroy

Sport et tourisme, considérés séparément, constituent des domaines importants de l'usage contemporain du temps de loisir; combinés, ils forment une pratique à part entière, le tourisme sportif, qui se singularise notamment par des lieux et des terrains de pratique, des rapports à l'espace sensibles comme discursifs, des formes de mobilité. C'est là l'objet de cette thèse: saisir l'originalité de ce phénomène de loisir dans ses dimensions spatiales. En tant qu'objet d'étude, il s'inscrit dans plusieurs enjeux et débats actuellement importants pour la géographie. Il touche à l'accroissement et à la diversification des mobilités, ainsi qu'à leur valorisation sociale et hédonique tendances qui ont conduit les sciences sociales à effectuer un "tournant mobilitaire ", c'est-à-dire à reconnaître le mouvement comme une modalité centrale de l'organisation des sociétés (Sheller et Urry, 2006). Le tourisme sportif témoigne également de l'hybridation progressive des loisirs avec les différents domaines et espaces de la vie; une géographie des pratiques, centrée sur l'implication de l'espace dans l'action (Lussault et Stock, 2010), permet de reconnaitre et d'étudier ce type de recompositions, de comprendre comment des schémas d'action font sens pour les individus et les groupes. Enfin, le tourisme sportif implique des émotions et affects relatifs à l'espace; et c'est en s'inscrivant dans le courant des "géographies émotionnelles " (Anderson et Smith, 2001) que l'on peut aborder efficacement des éléments tels que l'appréciation du mouvement et de l'action sportive, ou encore le fort 
investissement des espaces non urbains, dits "de nature", par des pratiques et imaginaires de loisir. Certains sports se définissent par cette idée de la nature, et celleci contribue fortement à faire de ces sports des motifs de voyage (Bourdeau, 2003) ; c'est pourquoi cette thèse se concentre sur les pratiques touristico-sportives de nature.

J'interroge ces pratiques autour de deux questions principales : celle du rôle de l'espace dans la constitution du commun, du lien social, et celle de l'appréciation des spatialités - entendues comme les diverses manières d'agir avec l'espace et de le considérer. Deux concepts fondamentaux permettent le traitement de ces questions: celui de communautés de pratiques, qui qualifie les ensembles d'individus partageant des schémas cohérents d'action et de compréhension; et celui d'engagement, qui désigne la coordination entre un projet d'action et l'environnement dans lequel il se déroule. Le cadre théorique général de cette thèse, dans lequel s'inscrivent ces concepts, est celui des théories de la pratique, représentées principalement par Theodore Schatzki (2002), pour sa théorie de l'organisation des pratiques sociales, et par Laurent Thévenot (2006), pour sa théorie des modes d'engagement et de coordination. En centrant l'analyse sur l'action, sur le corps et sur les modes communs de connaissance du monde, les théories de la pratique ont pour ambition de dépasser les dichotomies traditionnelles entre la structure et la liberté d'action, l'individu et le groupe, la matérialité et les conceptions. Pour l'objet d'étude de cette thèse, cela signifiait notamment accorder autant d'importance à la dimension corporelle et matérielle du tourisme sportif de nature qu'à sa dimension idéelle et représentationnelle.

3 Le travail est fondé empiriquement sur des enquêtes qualitatives auprès de personnes voyageant pour trois sports de nature, dans quelques-uns des lieux les plus réputés, les plus désirables et les plus attractifs pour ces sports : le parapente (à Annecy et à SaintAndré-les-Alpes, en France), le kayak de rivière (dans le haut bassin de la Durance, en France) et l'escalade sportive (dans le Verdon, en France, et à Kalymnos, en Grèce). Ces enquêtes ont abouti à 76 entretiens semi-directifs, avec 110 personnes, mais aussi à la collecte d'un corpus de photographies commentées de pratiquantes et pratiquants, donnant un aperçu de la valorisation personnelle par l'image de l'expérience touristicosportive. Des observations de terrain ainsi que des analyses de médias spécialisés (presse et sites web) viennent compléter ponctuellement le matériau empirique. Sur ces bases, la thèse présente et discute ses résultats en quatre chapitres, qui correspondent à quatre dimensions essentielles des spatialités du tourisme sportif de nature.

Une première analyse porte sur les espaces communs de la pratique touristico-sportive, espaces construits par les circulations et les ancrages, par les appropriations symboliques et physiques de certains lieux. À l'échelle mondiale, l'espace des pratiques touristico-sportives apparaît comme une «lecture» du monde sélective, hiérarchisante, qui se concrétise dans des mobilités guidées par un projet spécifique de loisir - phénomène que je qualifie de mondialisation affinitaire. Les médias spécialisés façonnent et valident cette hiérarchisation, en entretenant des conceptions mythiques et esthétiques des lieux; ils incitent également au voyage en véhiculant des valeurs de découverte, d'exotisme et d'aventure. Les lieux fonctionnent comme des centralités hautement spécialisées : ils sont définis dans leurs métriques mêmes par les modalités de chaque pratique touristico-sportive, comme je le montre avec la région de la HauteDurance, centralité mondiale pour les sports de montagne, accueillant en son sein une multitude de centralités de dimensions différentes aux pouvoirs d'attraction différents. 
La centralité des lieux tient aussi à leur capacité à rassembler physiquement la communauté de pratique, et ainsi à incarner les attraits de l'échange cosmopolite, de la connivence et des émotions partagées. Les pratiques touristico-sportives permettent donc d'éclairer la notion de centralité sous le jour de la spécialisation et de souligner le pouvoir d'organisation spatiale des communautés de pratique, y compris de loisir, à l'échelle globale comme locale.

Une seconde analyse explore le rôle de l'information géographique dans la coordination des pratiques touristico-sportives de nature. Cette coordination s'appuie sur des outils reprenant certaines fonctions des traditionnels « topoguides» des sports de nature, mais en les élargissant considérablement par la numérisation et la mise en ligne de l'information. Les plates-formes web collaboratives (en l'occurrence: CamptoCamp, RiverMap, EauxVives.Org, la Coupe Fédérale de Distance de vol libre et ThermalMaps) sont des modes de partage d'une information géographique ciblée et très spécialisée, qui contribuent ainsi à la constitution de savoirs spatiaux experts. Ceux-ci s'inscrivent plus généralement dans des langages de formalisation des spatialités complexes en jeu dans la pratique des terrains sportifs de nature, que je qualifie de jargons géographiques. Ces outils sont essentiels à la mise en commun des spatialités, à l'établissement de "grilles de lecture " partagées des environnements de pratique. La médiation numérique de ces spatialités ouvre en outre d'importantes possibilités pour cette mise en commun, en la rendant plus fluide, plus dynamique et plus collaborative. Elle permet, par exemple, une lecture fine des conditions météorologiques ou de terrain, et la mise à jour régulière de cette lecture.

Dans un troisième temps, je mène une réflexion sur l'appréciation de la spatialité en jeu dans les pratiques touristico-sportives de nature, en me concentrant sur la dimension corporelle de cette appréciation. Les discours des personnes interrogées, notamment au sujet de leur goût pour le voyage sportif et de leur appréciation des environnements de pratique, dessinent un mode d'engagement spécifique, que je qualifie d'immersionaction. Sont en effet fortement valorisées la stimulation du corps par l'effort; la focalisation du corps et de la conscience; les expériences de l'espace vécues comme originales et intenses; et la contemplation d'un environnement envisagé comme paysage ou comme nature. Le tourisme sportif de nature apporte une perspective originale sur les spatialités corporelles, en donnant à voir, avec l'immersion-action, une spatialité réflexive, une "spatialité d'attention à la spatialité ». Mon analyse étaye également l'idée selon laquelle la catégorie de nature est avant tout un rapport du corps à l'espace, qui trouve des traductions symboliques et esthétiques, notamment dans une conception relationnelle du paysage. Je décris enfin la pratique sportive de nature comme une mise en action de schémas corporels dans un environnement considéré comme un ensemble de "prises»; ce faisant, je montre comment la spatialité corporelle est travaillée et partagée par les individus, notamment en vue des satisfactions de la maitrise pratique, de la coordination harmonieuse du corps avec l'environnement biophysique, idéal structurant pour les communautés des sports de nature.

7 Dans un quatrième et dernier temps, cette thèse interroge le tourisme sportif de nature comme projet individuel autour de la notion d'engagement. Je montre ainsi les différentes définitions subjectives, spatiales, temporelles de la pratique de voyager pour le sport. Cette pratique couvre des espaces d'extension globale pour certaines personnes, régionale pour d'autres, pouvant accueillir dans les deux cas des pratiques 
intensives de l'espace en termes de densité et de régularité des mobilités. La relation entre voyage et sport ne fait aucun doute pour la grande majorité des personnes interrogées : elles voyagent bel et bien pour le sport; la pratique sportive organise nettement leur mobilité et leur séjour, à des degrés divers d'exclusivité toutefois. Cela se traduit dans les temporalités de la mobilité touristique, dominées par la pratique sportive ; mais également dans les temporalités générales du loisir, voire de la vie chez certains individus, pour lesquels le sport comme le tourisme sportif peuvent prendre une place considérable. La pratique touristico-sportive de nature constitue donc un mode à part entière d'engagement, dans la mesure où elle forme des schémas d'action cohérents relatifs à des aspirations relativement précises. Je propose de considérer l'engagement dans la mobilité pour le sport selon un gradient d'intensité, entre tourisme et mode de vie. Si le premier pôle, celui du tourisme, reste largement dominant, l'engagement fort de nombre de personnes dans cette pratique de loisir bien qu'il ne constitue que dans de rares cas un véritable mode de vie - incite à envisager la possibilité du tourisme en tant qu'extension, ou exportation, des engagements de loisir habituels, et non plus seulement en tant que rupture avec les activités du quotidien.

8 Cette thèse propose donc une exploration approfondie des spatialités d'un groupe social défini par des pratiques. Ce faisant, elle participe au renouvellement de la compréhension du rôle de l'espace dans la formation de la société, en montrant que des formes de communauté émergent dans les espaces-temps du hors-quotidien, dans la mobilité, et dans le partage de schémas corporels et sensibles d'appréhension de l'environnement - autant d'objets que la géographie intègre progressivement. Ma thèse propose également une analyse de ce que la passion fait au tourisme: elle montre comment des engagements forts de loisir peuvent brouiller les frontières entre quotidien et hors-quotidien, tout en contestant l'idée d'une "dé-différenciation " (Lash et Urry, 1994) généralisée du tourisme au sein des pratiques sociales. Elle propose enfin, dans le cadre des théories de la pratique (et en particulier en complément de Thévenot, 2006), de considérer le loisir comme un engagement à part entière ; un engagement fondé sur l'appréciation individuelle, sur les satisfactions hédoniques, mais où se déploient également des schémas d'action partagés, jusqu'à former, dans certains cas, des liens forts au sein de communautés de pratique.

\section{BIBLIOGRAPHIE}

Kay ANDERSON et SUsan J. SMITH, « Editorial: Emotional geographies », Transactions of the Institute of British Geographers, vol. 26, n 1, 2001.

Philippe BOURDEAU, Territoires du hors-quotidien : une géographie culturelle du rapport à l'ailleurs dans les sociétés urbaines contemporaines ; le cas du tourisme sportif de montagne et de nature, Université Joseph-Fourier-Grenoble 1, 2003.

Scott LASH et John URRY, Economies of signs and space, Sage, 1994. 
Michel LuSSAULT et Mathis STOCK, « 'Doing with space': towards a pragmatics of space », Social Geography, vol. 5, $\mathrm{n}^{\circ}$ 1, 2010.

Theodore R. SCHATZKI, The site of the social: a philosophical account of the constitution of social life and change, Pennsylvania State University Press, 2002

Mimi SHELleR et John URRY, « The New Mobilities Paradigm », Environment and Planning A, vol. 38, $n^{\circ} 2,2006$

Laurent THÉVENOT, L'action au pluriel : sociologie des régimes d'engagement, La Découverte, 2006.

\section{AUTEUR}

VALÉRIAN GEFFROY

vgeffroy[at]unistra.fr 